\title{
Supplementary Information for \\ Effects of surface electron doping and substrate on the superconductivity of epitaxial FeSe films
}

\author{
W. H. Zhang ${ }^{\dagger}$, X. Liu ${ }^{\dagger}$, C. H. P. Wen ${ }^{\dagger}$, R. Peng ${ }^{\dagger}$, S. Y. Tan ${ }^{\dagger}$, B. P. Xie ${ }^{\dagger, \dagger}$, \\ T. Zhang ${ }^{* \dagger, \dagger}$ D. L. Feng ${ }^{\dagger, *}$
}

${ }^{\dagger}$ State Key Laboratory of Surface Physics, Department of Physics, and Advanced Materials Laboratory, Fudan University, Shanghai 200433, China

${ }^{*}$ Collaborative Innovation Center of Advanced Microstructures, Fudan University, Shanghai 200433, China

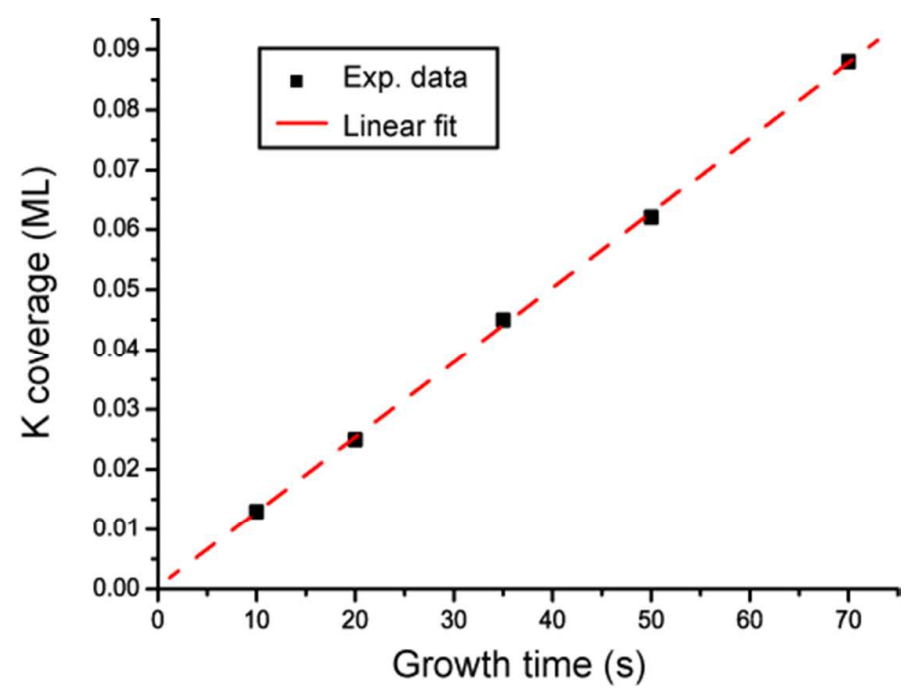

Fig. s1. The calibration of $\mathrm{K}$ growth rate. Black dots are measured $\mathrm{K}$ coverage vs. growth time. Red dashed line is the linear fit, which yields a growth rate of 0.075 $\mathrm{ML} / \mathrm{min}$. 


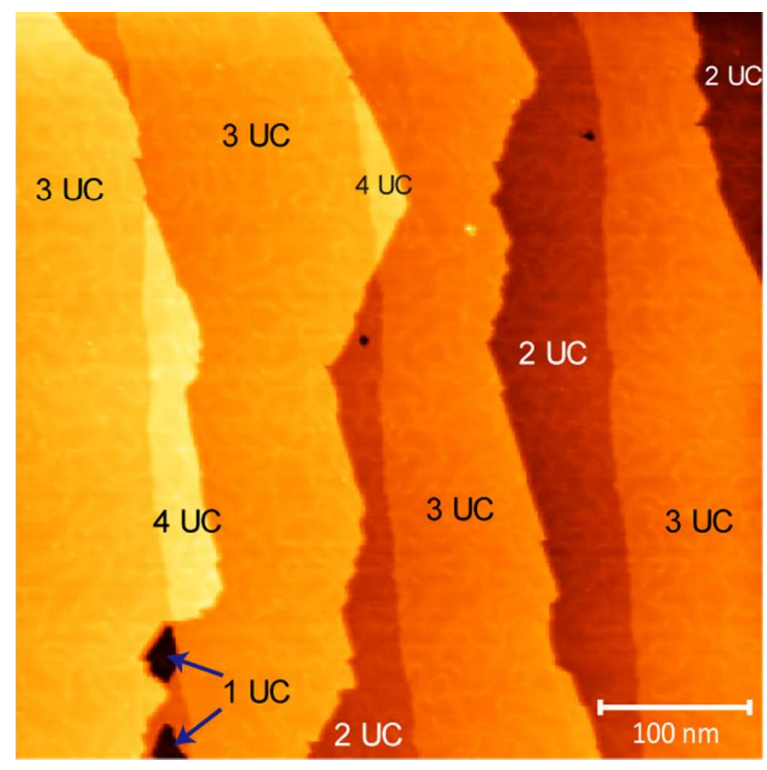

Fig. s2. Large scale STM topography of a FeSe/STO film with nominal thickness of 2.8 UC. Regions with different thickness from $1 \mathrm{UC}$ to $4 \mathrm{UC}$ coexist in the sample (1 UC regions are indicated by the arrows). Therefore the thickness dependence of the $\mathrm{SC}$ gap can be measured in the same sample with the same K coverage. 
2 UC FeSe/STO, Kc=0.045 ML

(a)

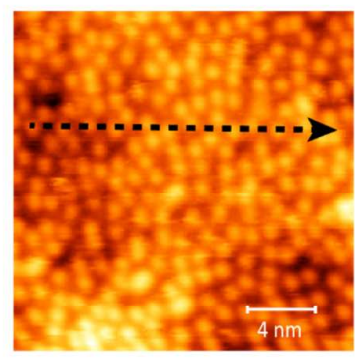

(d)

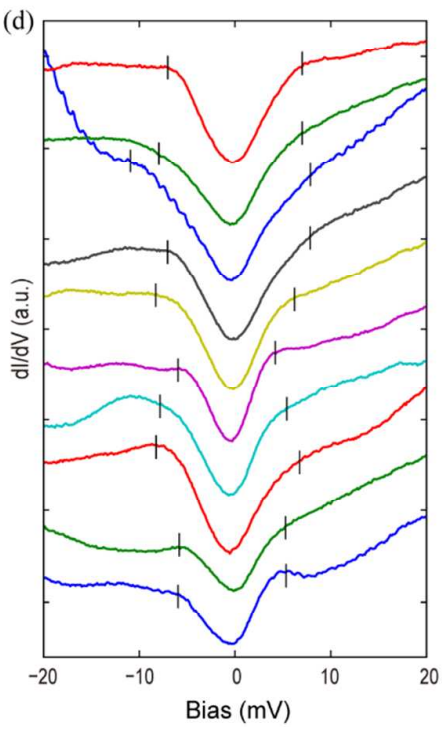

3 UC FeSe/STO, Kc=0.062 ML

(b)

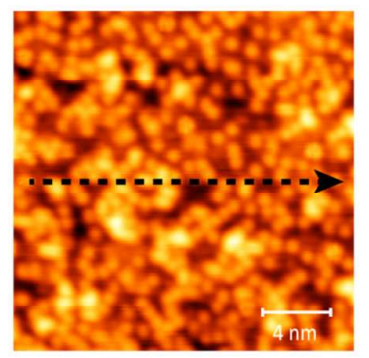

(e)

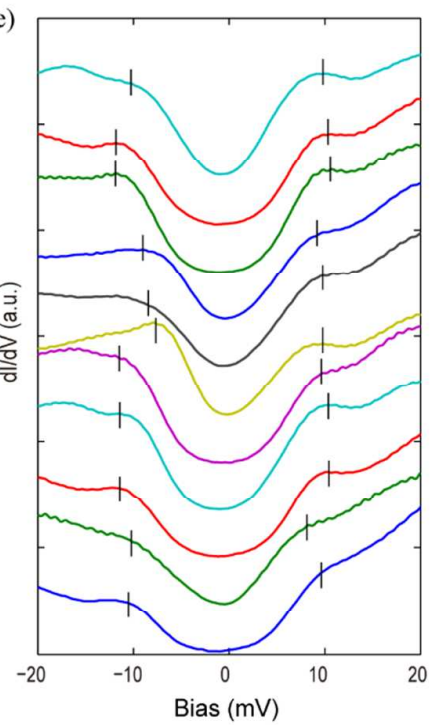

$4 \mathrm{UC} \mathrm{FeSe} / \mathrm{STO}, \mathrm{Kc}=0.088 \mathrm{ML}$

(c)
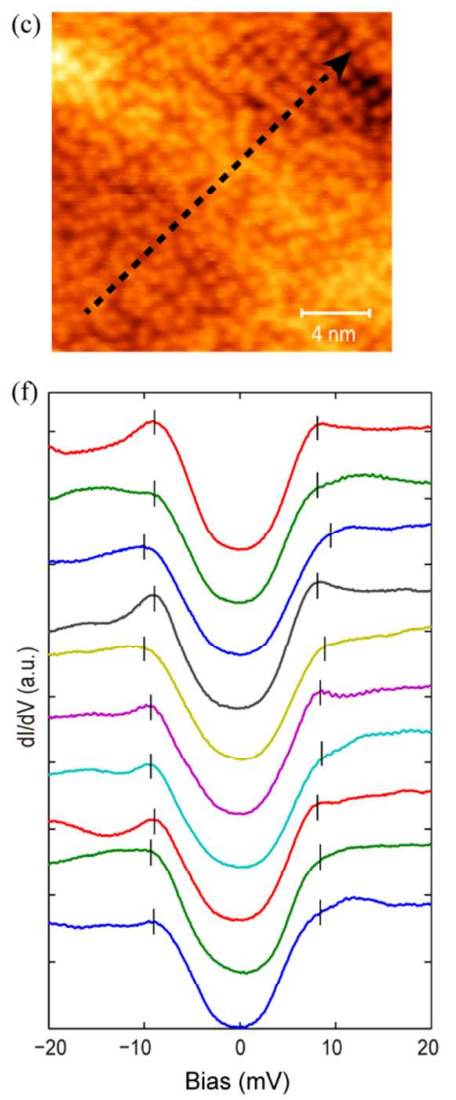

Fig. s3. Spatial inhomogeneity of the SC gap in $\mathbf{K}$ dosed FeSe. (a c): STM images of $2 \mathrm{UC}, 3 \mathrm{UC}$ and $4 \mathrm{UC} \mathrm{FeSe} / \mathrm{STO}$, with Kc of $0.045 \mathrm{ML}, 0.062 \mathrm{ML}$ and 0.088 ML, respectively. (d f f): dI/dV spectra taken along the line cut marked in (a), (b) and (c), respectively. The short bars indicate the position of the coherence peaks or kinks near the gap edge. The gap magnitude is determined by one half of the energy between two coherence peaks or kinks. 


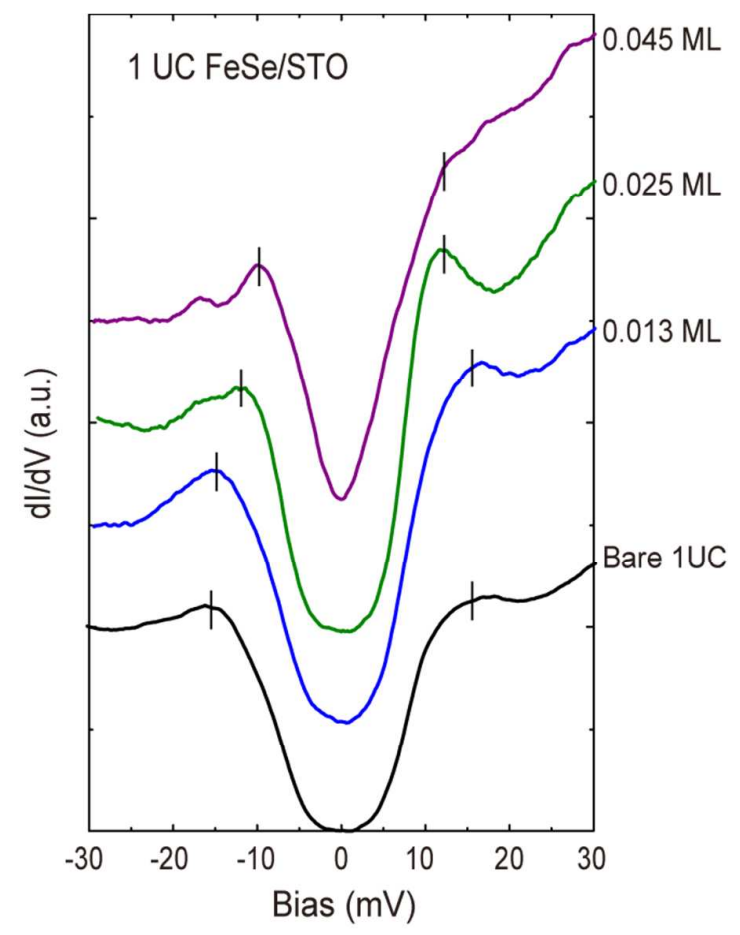

Fig. s4. The K dosing dependence of the SC gap of 1UC FeSe/STO.: The short bars indicate the position of the coherence peaks 